Journal of Bangladesh Academy of Sciences, Vol. 36, No. 2, 159-170, 2012

\title{
A CHAOTIC THREE DIMENSIONAL NON-LINEAR AUTONOMOUS SYSTEM BEYOND LORENZ TYPE SYSTEMS
}

\author{
MD. SHARIFUL ISLAM KHAN* AND MD. SHAHIDUL ISLAM ${ }^{1}$ \\ Department of Mathematics, National University, Gazipur-1704, Bangladesh
}

\begin{abstract}
Some fundamental properties of a chaotic three-dimensional non-linear system of the Lorenz type systems were studied. The invariance, dissipation, bifurcation and the strange attractors were investigated and analyzed one 1-scroll, two 2-scroll and two 4-scroll attractors by adding control parameters to this system. The relationship and connecting function for the 2-scroll attractor of this system were also explored.
\end{abstract}

Key words: Chaos, Lorenz model, Bifurcation, Attractor, Strange attractors

\section{INTRODUCTION}

It came as a big surprise to most scientists when Lorenz discovered chaos in a simple system of three-dimensional quadratic autonomous ordinary differential equations in 1963. For nearly 50 years, many simple chaotic flows have been found and further studied within the framework of three-dimensional quadratic autonomous systems. These simple chaotic systems have stimulated a great deal of interest in the studies of chaotic dynamics from a unified point of view, as well as related chaos control and synchronization problems.

Recently, chaos has been found to be very useful and has great potential in many technological disciplines such as in information and computer science, power systems protection, biomedical systems analysis, flow dynamics and liquid mixing, encryption and communications, and so on (Lü et al. 2002). Therefore, academic research on chaotic dynamics has evolved from the traditional trend of analyzing and understanding chaos to the new direction of controlling and utilizing it (Ott et al. 1990). In a broader sense, chaos control can be divided into two categories: one is to suppress the chaotic dynamical behavior when it is harmful and the other is to create or enhance chaos when it is desirable - known as chaotification or anti-control of chaos (Lü et al. 2002). Very recently, there has been increasing interest in exploiting chaotic dynamics in engineering applications, where some attention has been focused on effectively creating chaos via simple physical systems such as electronic circuits (Wang and Chen 2000) and switching piecewise-linear controllers.

* Corresponding author: <mshahidul11@yahoo.com>.

${ }^{1}$ Department of Mathematics, University of Dhaka, Dhaka-1000, Bangladesh. 
The authors studied three-dimensional quadratic autonomous chaotic system, which can display two 1-scroll or two complex 2-scroll chaotic attractors simultaneously. They also introduced a new chaotic system and investigated the dynamical behaviors of this chaotic system, by employing some tools used in Chen and Ueta (1999). Here in the compound structure of chaotic attractors was analyzed for the two 2-scroll attractors of this new system.

\section{GENERAL LORENZ SYSTEM FAMILY}

For three-dimensional autonomous systems (Čelikovskỳ and Chen 2002) introduced a classification in terms of $a_{12} a_{21}$ where $a_{12}$ and $a_{21}$ are the corresponding entries of the linear part of the system described by the constant matrix

$$
A=\left\lfloor a_{i j}\right\rfloor_{3 \times 3} .
$$

Historically, the Lorenz system of equations is perhaps the first of the non-linear dynamical systems found to exhibit sensitive dependence on initial conditions and chaos. The Lorenz system is described by the following non-linear differential equations;

$$
\left.\begin{array}{l}
\dot{x}=\sigma(y-x) \\
\dot{y}=r x-y-x z \\
\dot{z}=x y-b z
\end{array}\right\}
$$

Typical parameters for a Lorenz system are $\sigma=10, r=28$ and $b=8 / 3$. According to the form of the generalized Lorenz system (Čelikovskỳ and Chen 2002), we have

$$
\left[\begin{array}{c}
\dot{x} \\
\dot{y} \\
\dot{z}
\end{array}\right]=\left[\begin{array}{ccc}
a_{11} & a_{12} & 0 \\
a_{21} & a_{22} & 0 \\
0 & 0 & a_{33}
\end{array}\right]\left[\begin{array}{l}
x \\
y \\
z
\end{array}\right]+x \cdot\left[\begin{array}{ccc}
0 & 0 & 0 \\
0 & 0 & -1 \\
0 & 1 & 0
\end{array}\right]\left[\begin{array}{l}
x \\
y \\
z
\end{array}\right]
$$

The Lorenz system is described by

$$
\begin{aligned}
& {\left[\begin{array}{c}
\dot{x} \\
\dot{y} \\
\dot{z}
\end{array}\right]=\left[\begin{array}{ccc}
-\sigma & \sigma & 0 \\
r & -1 & 0 \\
0 & 0 & -b
\end{array}\right]\left[\begin{array}{l}
x \\
y \\
z
\end{array}\right]+x \cdot\left[\begin{array}{ccc}
0 & 0 & 0 \\
0 & 0 & -1 \\
0 & 1 & 0
\end{array}\right]\left[\begin{array}{l}
x \\
y \\
z
\end{array}\right]} \\
& {\left[\begin{array}{c}
\dot{x} \\
\dot{y} \\
\dot{z}
\end{array}\right]=\left[\begin{array}{ccc}
-10 & 10 & 0 \\
28 & -1 & 0 \\
0 & 0 & -8 / 3
\end{array}\right]\left[\begin{array}{l}
x \\
y \\
z
\end{array}\right]+x \cdot\left[\begin{array}{ccc}
0 & 0 & 0 \\
0 & 0 & -1 \\
0 & 1 & 0
\end{array}\right]\left[\begin{array}{c}
x \\
y \\
z
\end{array}\right]}
\end{aligned}
$$

The Lorenz system satisfies $a_{12} \cdot a_{21}>0, \quad[10 \cdot 28>0]$.

Chen constructed another chaotic system from an engineering feedback control approach (Lorenz 1963). 
Chen's system is of the following form:

$$
\left.\begin{array}{l}
\dot{x}=\sigma(y-x) \\
\dot{y}=(r-\sigma) x-r y-x z \\
\dot{z}=x y-b z
\end{array}\right\}
$$

Typical parameters for the Chen system are $\sigma=35, r=28$, and $b=3$. According to the generalized Lorenz system form, the Chen system is described by

$$
\left[\begin{array}{c}
\dot{x} \\
\dot{y} \\
\dot{z}
\end{array}\right]=\left[\begin{array}{ccc}
-35 & 35 & 0 \\
-7 & 28 & 0 \\
0 & 0 & -3
\end{array}\right]\left[\begin{array}{l}
x \\
y \\
z
\end{array}\right]+x \cdot\left[\begin{array}{ccc}
0 & 0 & 0 \\
0 & 0 & -1 \\
0 & 1 & 0
\end{array}\right]\left[\begin{array}{c}
x \\
y \\
z
\end{array}\right]
$$

The Chen system satisfies $a_{12} \cdot a_{21}<0, \quad[35 \cdot(-7)<0]$.

Lü and Chen (2002) found a critical new chaotic system, which is generated by the following three-dimensional autonomous system:

$$
\left.\begin{array}{l}
\dot{x}=\sigma(y-x) \\
\dot{y}=r y-x z \\
\dot{z}=x y-b z
\end{array}\right\}
$$

Typical parameters for the Lü system are $\sigma=36, r=20$, and $b=3$. This system bridges the gap between the Lorenz and Chen systems.

According to the form of generalized Lorenz system, the Lü system is described by

$$
\left[\begin{array}{c}
\dot{x} \\
\dot{y} \\
\dot{z}
\end{array}\right]=\left[\begin{array}{ccc}
-36 & 36 & 0 \\
0 & 20 & 0 \\
0 & 0 & -3
\end{array}\right]\left[\begin{array}{l}
x \\
y \\
z
\end{array}\right]+x \cdot\left[\begin{array}{ccc}
0 & 0 & 0 \\
0 & 0 & -1 \\
0 & 1 & 0
\end{array}\right]\left[\begin{array}{l}
x \\
y \\
z
\end{array}\right]
$$

The Lü system satisfies $a_{12} \cdot a_{21}=0, \quad[36 \cdot 0=0]$.

\section{A NEW CHAOTIC SYSTEM AND ITS ANALYSES}

Consider the following simple three-dimensional quadratic autonomous system, which can display two chaotic attractors simultaneously:

$$
\left.\begin{array}{rl}
\dot{x} & =-\frac{\alpha \beta}{\alpha+\beta} x-y z \\
\dot{y} & =x z+\alpha y \\
\dot{z} & =x y+\beta z
\end{array}\right\}
$$

where $\alpha, \beta$ are real constants. This system is found to be chaotic in a wide parameter range and has many interesting complex dynamical behaviors. 
According to the form (B), the Lorenz system (A) satisfies

$$
a_{12} \cdot a_{21}>0
$$

the Chen system (E) satisfies

$$
a_{12} \cdot a_{21}<0,
$$

the Lü system (G) satisfies

$$
a_{12} \cdot a_{21}=0,
$$

and the new system (1) also satisfies

$$
a_{12} \cdot a_{21}=0,
$$

The system (1) has similar but different dynamical behaviors with the transition system. By a suitable non-singular transform, it is assumed without loss of generality that $A=\left\lfloor a_{i j}\right\rfloor_{3 \times 3}=\operatorname{diag}\{a, b, c\}$. In this setting, Lü and Chen (2002) derived another classification condition in terms of $a b+b c+c a$ for the three-dimensional quadratic autonomous systems. Using this condition, the 4-scroll system satisfies

$$
a b+b c+c a \neq 0
$$

while system (1) here satisfies

$$
a b+b c+c a=0
$$

Therefore, system (1) is a new and particular system that satisfies two different classification conditions as described above. Moreover, this system has many interesting complex dynamical behaviors, as will be seen below.

\section{DYNAMICAL BEHAVIORS OF THE SYSTEM}

The new system (1) shares various properties with some known three-dimensional quadratic autonomous systems, such as the Lorenz system. These are described in the following:

Symmetry and invariance: First, note that the system is invariance under the transforms $(x, y, z) \rightarrow(x,-y,-z),(x, y, z) \rightarrow(-x,-y, z)$, and $(x, y, z) \rightarrow(-x, y,-z)$. That is, system (1) is symmetrical about the three coordinate axes $x, y, z$, respectively.

Dissipation and existence of attractor: The rate of the volume contraction for the system (1) is given by the Lie derivative

$$
\frac{1}{V} \frac{d V}{d t}=\frac{\partial \dot{x}}{\partial x}+\frac{\partial \dot{y}}{\partial y}+\frac{\partial \dot{z}}{\partial z}
$$




$$
\begin{aligned}
\frac{1}{V} \frac{d V}{d t} & =-\frac{\alpha \beta}{\alpha+\beta}+\alpha+\beta \\
& =\frac{\alpha^{2}+\alpha \beta+\beta^{2}}{\alpha+\beta}=\frac{\left(\alpha+\frac{1}{2} \beta\right)^{2}+\frac{3}{4} \beta^{2}}{\alpha+\beta} . \\
\Rightarrow V(t) & =V_{0} e^{\frac{\alpha^{2}+\alpha \beta+\beta^{2}}{\alpha+\beta} t} .
\end{aligned}
$$

It follows that the volume of a bounded region of this system will decrease with $e^{\frac{a^{2}+\alpha \beta+\beta^{2}}{a+b}}$

volume. Thus the system is clearly dissipative. This is the well-known necessary condition for the asymptotic motion to settle on to an attractor.

Equilibria and Bifurcations: To find the equilibria of the system (1), let us consider $\dot{x}=\dot{y}=\dot{z}=0$.

If $\alpha \beta>0$ then system (1) has five equilibria:

$$
\begin{aligned}
& S_{1}=(0,0,0), S_{2}=\left(\sqrt{\alpha \beta},|\beta| \sqrt{\frac{\alpha}{\alpha+\beta}},-\alpha \sqrt{\frac{\beta}{\alpha+\beta}}\right), S_{3}=\left(\sqrt{\alpha \beta},-|\beta| \sqrt{\frac{\alpha}{\alpha+\beta}}, \alpha \sqrt{\frac{\beta}{\alpha+\beta}}\right) \\
& S_{4}=\left(-\sqrt{\alpha \beta},|\beta| \sqrt{\frac{\alpha}{\alpha+\beta}}, \alpha \sqrt{\frac{\beta}{\alpha+\beta}}\right), S_{5}=\left(-\sqrt{\alpha \beta},-|\beta| \sqrt{\frac{\alpha}{\alpha+\beta}},-\alpha \sqrt{\frac{\beta}{\alpha+\beta}}\right)
\end{aligned}
$$

If $\alpha \beta \leq 0$, then system (1) has a unique equilibrium $S_{1}=(0,0,0)$. Pitchfork bifurcation of the null solution at $\alpha=0$ (or $\beta=0$ ) can be observed, if $\beta$ (or $\alpha$ ) is fixed but $\alpha$ (or $\beta$ ) is varied.

Moreover, any two nonzero equilibria are symmetric about one of the axes $x, y, z$, that is,

(i) $S_{2}$ and $S_{3}, S_{4}$ and $S_{5}$ are symmetric with respect to the $x$-axis.

(ii) $S_{2}$ and $S_{4}, S_{3}$ and $S_{5}$ are symmetric with respect to the $y$-axis.

(iii) $S_{2}$ and $S_{5}, S_{3}$ and $S_{4}$ are symmetric with respect to the $z$-axis.

Linearizing the system (1) at $S_{1}$, we have three eigenvalues:

$$
\lambda_{1}=-\alpha \beta /(\alpha+\beta), \lambda_{2}=\alpha, \text { and } \lambda_{3}=\beta .
$$


Again, linearizing the system at $S_{2}, S_{3}, S_{4}, S_{5}$ yields the following same characteristic equation:

$$
f(\lambda)=\lambda^{3}-\frac{\alpha^{2}+\alpha \beta+\beta^{2}}{\alpha+\beta} \lambda^{2}-\frac{4 \alpha^{2} \beta^{2}}{\alpha+\beta}=0 .
$$

Investigation for Hopf bifurcation: Since the characteristic equation for equilibrium $S_{1}=(0,0,0)$ has three eigenvalues: $\lambda_{1}=-\alpha \beta /(\alpha+\beta), \lambda_{2}=\alpha$, and $\lambda_{3}=\beta$, which are not purely imaginary, so there is no Hopf bifurcation at $S_{1}$.

For equilibria $S_{2}, S_{3}, S_{4}, S_{5}$, we have the same characteristic equation (2), so only the equilibrium $S_{2}$ is discussed. If Hopf bifurcation appears at equilibrium $S_{2}$, then it may assume that two zeros are $\lambda= \pm w i$ for some real $w$, and the sum of the three zeros of the cubic polynomial $f$ is

$$
\lambda_{1}+\lambda_{2}+\lambda_{3}=\frac{\alpha^{2}+\alpha \beta+\beta^{2}}{\alpha+\beta} .
$$

Hence, $\lambda_{3}=\left(\alpha^{2}+\alpha \beta+\beta^{2}\right) /(\alpha+\beta)$. However

$$
f\left(\lambda_{3}\right)=f\left(\frac{\alpha^{2}+\alpha \beta+\beta^{2}}{\alpha+\beta}\right)=-\frac{4 \alpha^{2} \beta^{2}}{\alpha+\beta}=0 \Rightarrow \alpha \beta=0 .
$$

This contradicts with the condition $\alpha \beta>0$. Therefore, there is no Hopf bifurcation at $S_{2}$. Similarly, this is true for each $S_{3}, S_{4}, S_{5}$. So it can be said that Hopf bifurcation does not appear at any equilibrium $S_{i}(i=1,2, \cdots, 5)$ of the system (1).

\section{Comment:}

1. It is quite a strange phenomenon to have no Hopf bifurcation in a threedimensional quadratic autonomous chaotic system. All known threedimensional quadratic autonomous chaotic systems have Hopf bifurcations at some of their equilibria, such as in the Lorenz system, Chen system, transition system, unified chaotic system.

2. Since the coefficient of $\lambda$ in (2) is zero, equation (2) does not have two conjugate imaginary roots for any parameters $\alpha, \beta$. However, the coefficient of $\lambda$ in the characteristic equation is non-zero for most known three-dimensional quadratic autonomous chaotic systems such as those just mentioned.

\section{DYNAMICAL STRUCTURE OF THE NEW CHAOTIC SYSTEM}

When $\alpha=-10, \beta=-4$, this system can display two complex 2-scroll chaotic attractors, as shown in Fig. 1. According to their geometric locations, these two coexisting attractors are called upper and lower attractor here for convenience. Various projections of these attractors are shown in Fig. 2. 


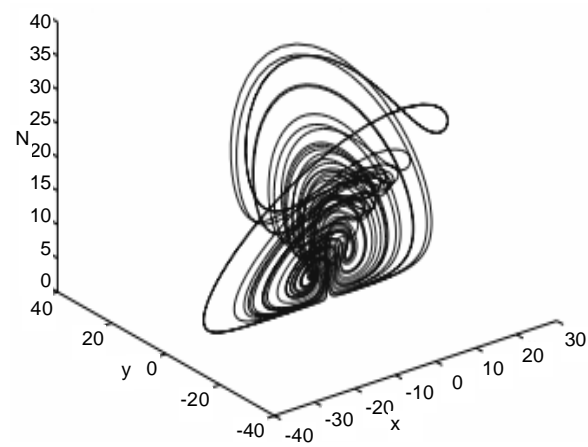

(a)

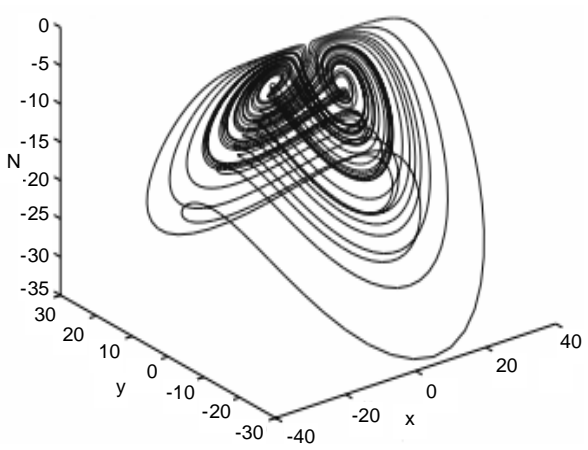

(b)

Fig. 1. Two coexisting 2-scroll chaotic attractors. (a) upper attractor, (b) lower attractor. $(\alpha=-10, \beta=-4)$.
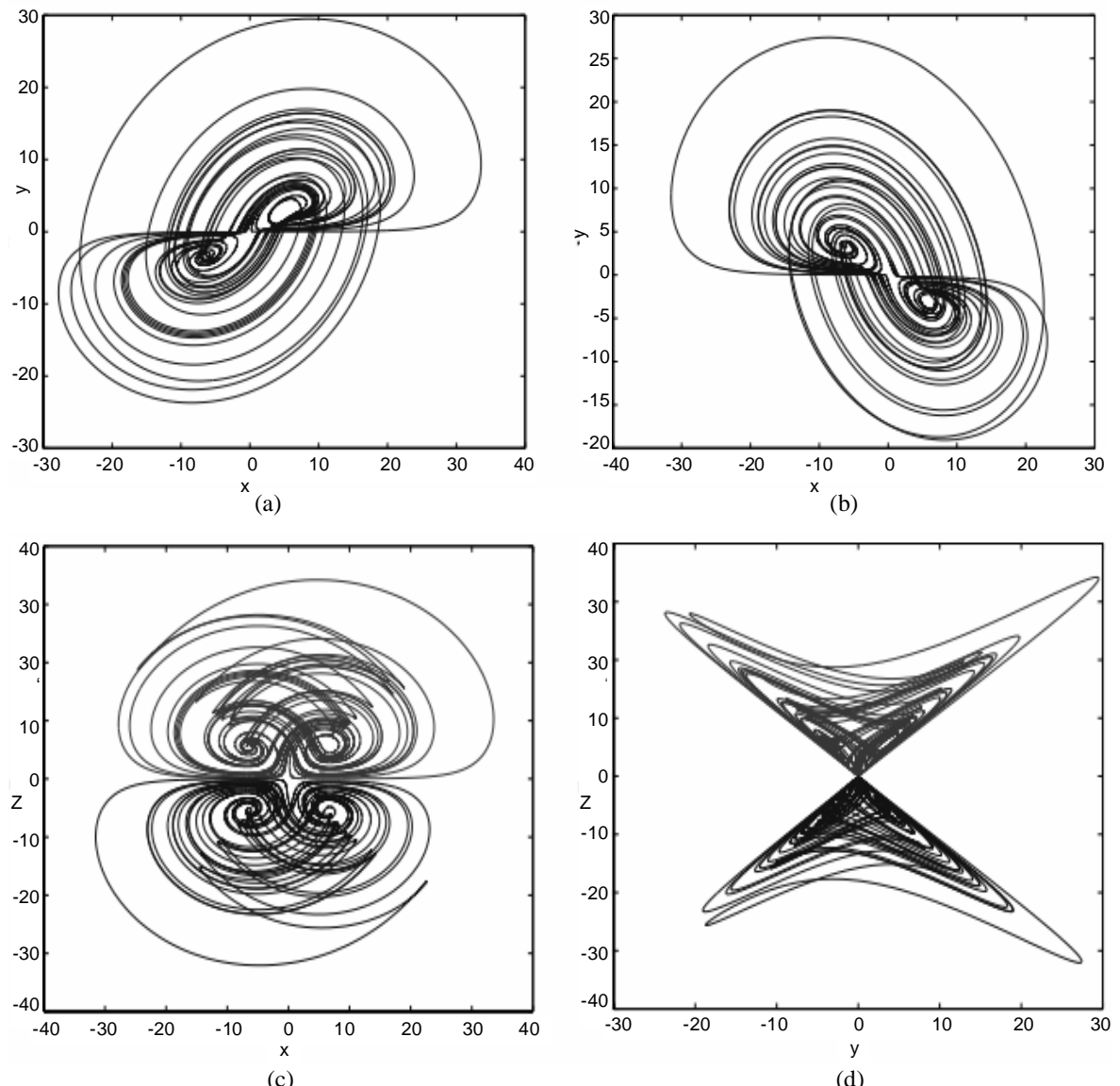

Fig. 2. Various projections of the upper and lower attractor. (a) $x-y$ (upper-attractor), (b) $x-y$ (lower-attractor), (c) $x-z$, (d) $y-z$. 
Compound structure of the upper and lower attractor: In order to investigate the compound structures of the upper and the lower-attractor, a constant control term is added to the first equation of the system (1)

$$
\left.\begin{array}{l}
\dot{x}=-\frac{\alpha \beta}{\alpha+\beta} x-y z+u \\
\dot{y}=x z+\alpha y \\
\dot{z}=x y+\beta z
\end{array}\right\}
$$

Assume that $\alpha=-10, \beta=-4$. When $u=9$, we obtain the right-attractors of the original upper and lower attractor, and their projections on the $x-z$ plane are shown in Fig. 3(a), (b); while when $u=-9$, one has their mirror images, i.e. the two left attractors and their projections on the $x-z$ plane, as shown in Fig. 3(c), (d).

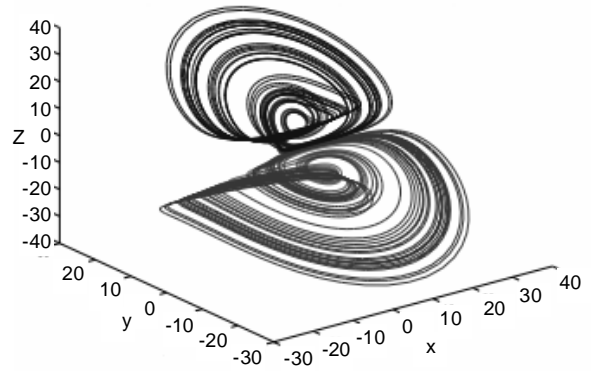

(a)

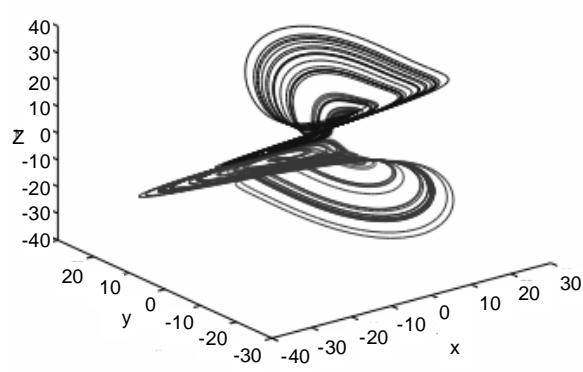

(c)

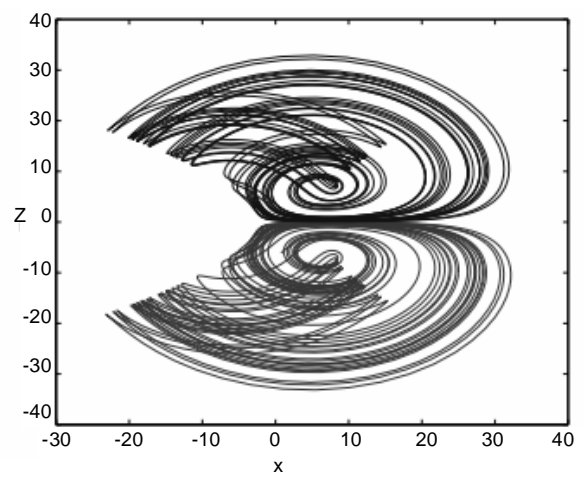

(b)

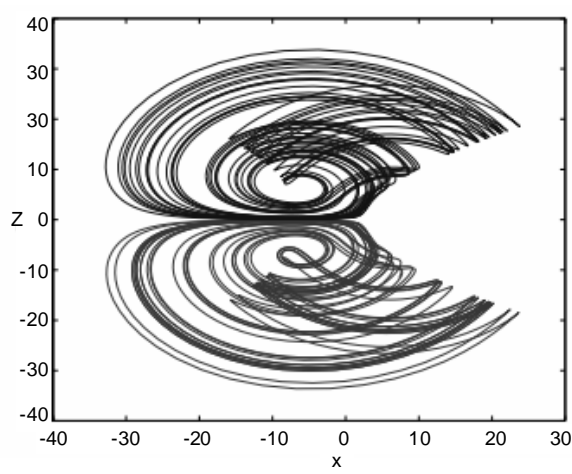

(d)

Fig. 3. The right- and left forming attractors and their projections, for the upper-attractor and lower-attractor. (a and b) $u=9$; (c and d) $u=-9$. 
Connecting the upper and lower attractor: As mentioned, system (1) has five equilibria: $S_{1}, S_{2}, S_{3}, S_{4}, S_{5}$ in which $S_{2}, S_{3}$ are above the plane $z=0$ while $S_{4}, S_{5}$ are below this plane. Furthermore, there is a close correlation between the equilibria $S_{1}, S_{2}, S_{3}$ and the upper attractor. Also, there is a close correlation between the equilibria $S_{1}, S_{4}, S_{5}$ and the lower attractor.

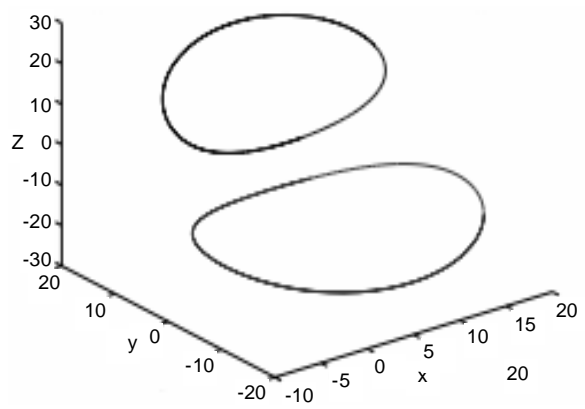

(a)

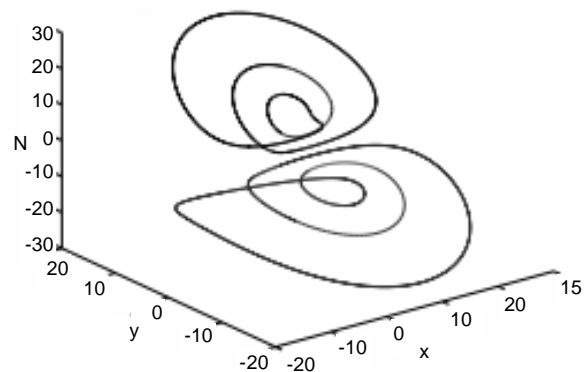

(c)

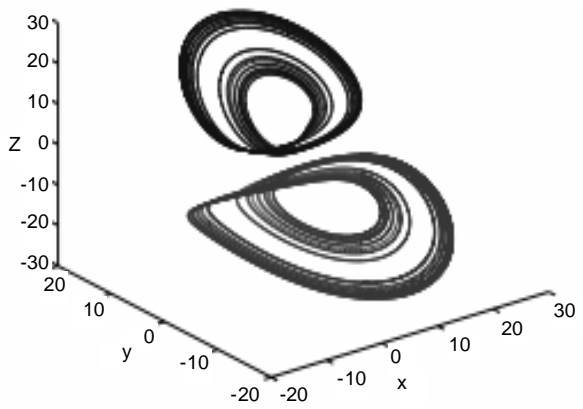

(b)

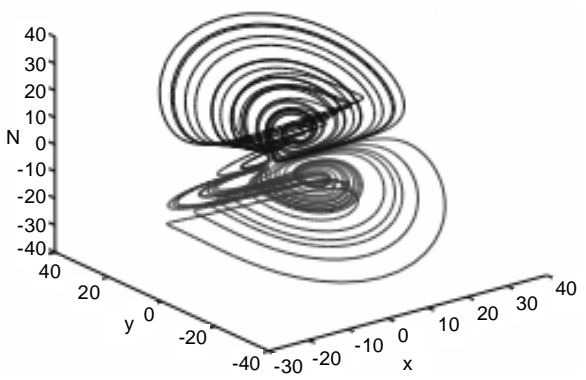

(d)

Fig. 4. Phase portraits of system (3). (a) $u=50$, (b) $u=19$, (c) $u=14.7$ and (d) $u=7$.

Specially, the upper and lower attractor are symmetric. It is therefore interesting to ask if there is a simple controller that can connect the upper and lower attractor. This section gives a positive answer to this question. In fact, a constant controller works well and can connect the upper and lower attractor to form a 4-scroll chaotic attractor from a 2 -scroll chaotic attractor.

In order to connect the upper and lower attractor, a simple constant controller $v$ is added to the second equation of the system (1), giving

$$
\left.\begin{array}{l}
\dot{x}=-\frac{\alpha \beta}{\alpha+\beta} x-y z \\
\dot{y}=x z+\alpha y+v \\
\dot{z}=x y+\beta z
\end{array}\right\}
$$


Let $\alpha=-10, \beta=-4$. When $v=5$, system (4) displays a 4-scroll chaotic attractor, as shown in Fig. 5.

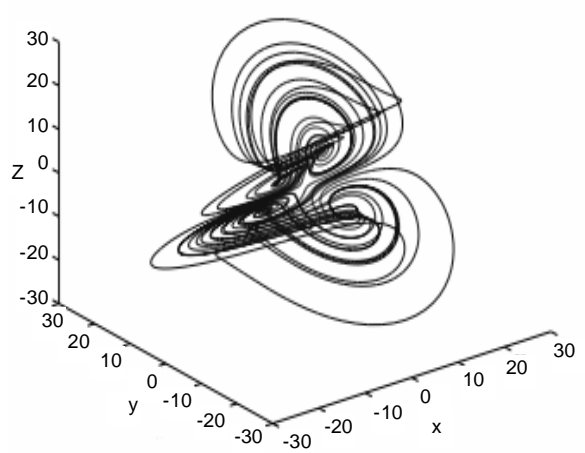

(a)

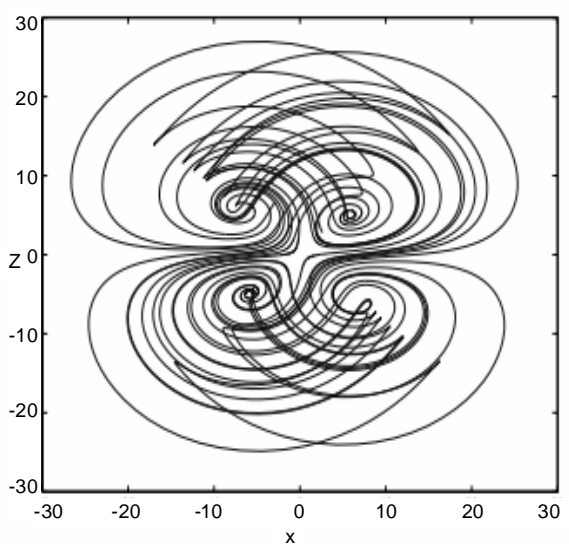

(c)

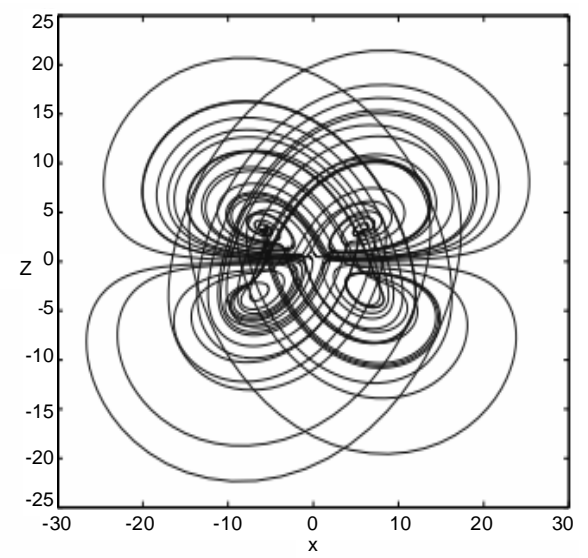

(b)

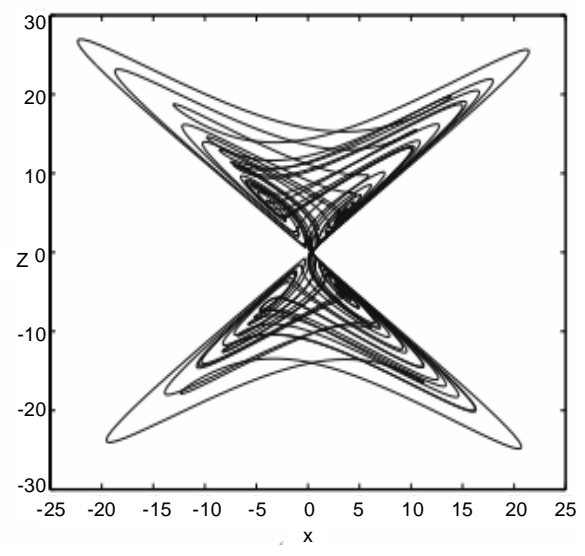

(d)

Fig. 5. The 4-scroll chaotic attractor of system (4) and its projections on various planes. (a) 3D phase portrait, (b) $x-y$ plane projection, (c) $x-z$ plane projection and (d) $y-z$ plane projection. $(\alpha=-10, \beta=-4, v=5$.)

Controlling in between the upper and lower attractor: As is known now, system (1) can display two 2-scroll chaotic upper and lower attractors. It is, therefore interesting to know the relationship between the upper and lower attractor, and if the two 2-scroll chaotic attractors can be confined to either one of them via a simple constant control. 
To do so, a constant controller $\mathrm{m}$ is added to the third equation of the system (1), so as to obtain

$$
\left.\begin{array}{l}
\dot{x}=-\frac{\alpha \beta}{\alpha+\beta} x-y z \\
\dot{y}=x z+\alpha y \\
\dot{z}=x y+\beta z+m
\end{array}\right\}
$$

When $\alpha=-10, \beta=-4, m=1$, system (5) displays a 2-scroll chaotic attractor upper-attractor - as shown in Fig. 6(a); when $m=-1$, the system shows another 2-scroll chaotic attractor - lower-attractor - as displayed in Fig. 6(b). Therefore, the original upper-attractor and lower attractor of the system (1) are confined to only one, either the upper or the lower attractor, via a simple constant control.

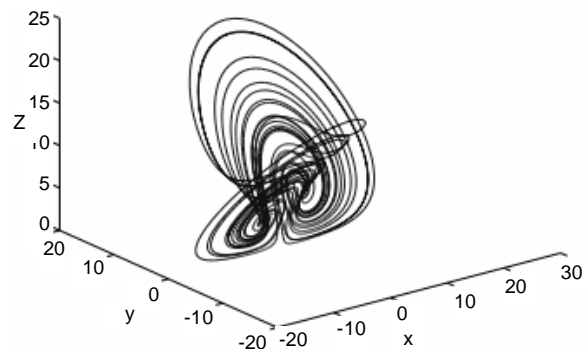

(a)

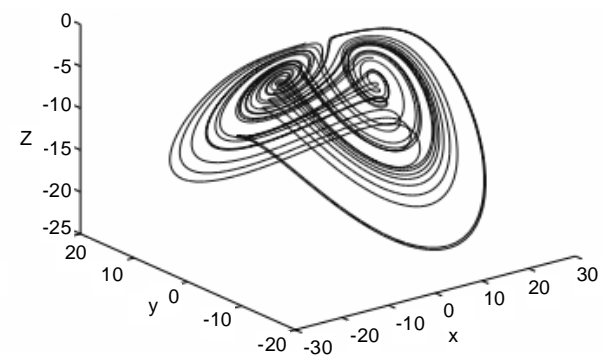

(b)

Fig. 6. The 2-scroll chaotic attractors of system (5). (a) $m=1$, (b) $m=-1 .(\alpha=-10, \beta=-4)$.

In fact, system (5) establishes a kind of connection between the upper and the lower attractor. When the controller $m$ is large, e.g. $m=1$, system (5) only displays the upperattractor; when the controller $m$ is small, e.g. $m=-1$, the system only shows the lowerattractor; when $m=0$, the system displays both.

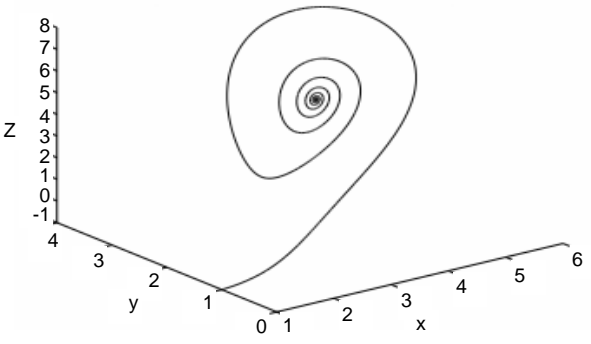

(a)

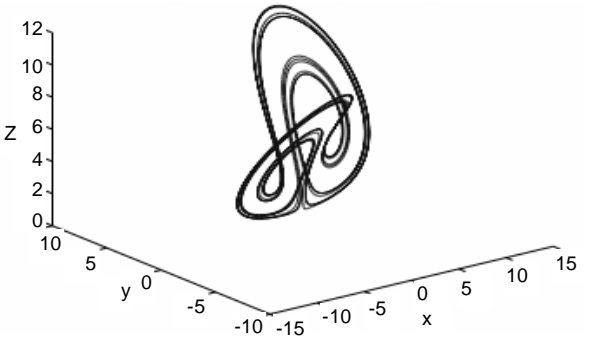

(b)

Fig. 7. Phase portraits of system (5). (a) $m=15$, (b) $\mathrm{m}=6.4$. $(\alpha=-10, \beta=-4$ ). 


\section{Comment:}

(1) When $|m|$ is large enough, e.g. $|m|>12.2$, the trajectories of system (5) converge to a point, as shown in Fig. 7(a); (ii) when $|\mathrm{m}|$ decreases gradually, there appear period-doubling bifurcations, as displayed in Fig. 7(b); (iii) when $|m|$ is relatively small, the upper and lower attractors are confined to a chaotic attractor, as shown in Fig. 6 and (iv) when $|m|$ is small enough, system (5) also has two 2-scroll attractors.

(2) For $m>12.2$, the trajectories of system (5) converge to a point above the plane $z=0$, while when $m<-12.2$, to a point below the plane $z=0$. Similarly, for $0.01<m<6.4$, system (5) is confined to an upper attractor, while when $-6.4<m<-0.01$, system (5) is confined to a lower attractor.

\section{CONCLUSION}

This article presented a new chaotic system of three-dimensional quadratic autonomous equations, which has generated two 2-scroll chaotic attractors simultaneously with five equilibriums. Dynamical behaviors of this new chaotic system, including some basic dynamical properties, invariance, dissipation, bifurcations, compound structures of the new attractors and their connections have been investigated both theoretically and numerically.

\section{REFERENCES}

Čelikovskỳ, S. and G. Chen. 2002. On a generalized Lorenz canonical form of chaotic systems, Inter. J. Bifurcation and Chaos 12: 1789-1812.

Chen, G. and T. Ueta. 1999. Yet another chaotic attractor. Inter. J. Bifurcation and Chaos 9: 1465-1466.

Lorenz, E. N. 1963. Deterministic nonperiodic flow. J. Atmos. Sci. 20: 130-141.

Lï, J. and G. Chen. 2002. A new chaotic attractor coined. Inter. J. Bifurcation and Chaos 12: 659-661.

Lü, J., J. Lu and S. Chen. 2002. Chaotic Time Series Analysis and Its Applications. Wuhan University Press, Wuhan, China.

Lü, J., T. Zhou, G. Chen and X. Yang. 2002. Generating chaos with a switching piecewise-linear controller. Chaos 12: 344-349.

Ott, E., C. Grebogi and J. A. Yorke. 1990. Controlling chaos. Physical Review Letter 64: 11961199.

Wang, X. and G. Chen. 1999. On feedback anticontrol of discrete chaos. Inter. J. Bifurcation and Chaos 9: 1435-1441.

Wang, X. and G. Chen. 2000. Chaotification via arbitrarily small feedback controls: Theory, method and applications. Inter. J. Bifurcation and Chaos 10: 549-570.

(Received revised manuscript on 14 September, 2012) 